\title{
High-pressure x-ray absorption and diffraction study of the self-doped superconductor $\mathrm{EuFBiS}_{2}$
}

\author{
E. Paris,,${ }^{1,2, *}$ B. Joseph $\odot,{ }^{3}$ C. Marini, ${ }^{4}$ K. Terashima, ${ }^{5}$ T. Wakita, ${ }^{5}$ T. Yokoya,${ }^{5}$ Y. Mizuguchi, ${ }^{6}$ T. Mizokawa,${ }^{7}$ and N. L. Saini ${ }^{1,}$ \\ ${ }^{1}$ Dipartimento di Fisica, Universitá di Roma “La Sapienza” - P. le Aldo Moro 2, 00185 Roma, Italy \\ ${ }^{2}$ Swiss Light Source, Photon Science Division, Paul Scherrer Institut, 5232 Villigen PSI, Switzerland \\ ${ }^{3}$ Elettra Sincrotrone Trieste, Strada Statale 14, Km 163.5, Basovizza, 34149 Trieste, Italy \\ ${ }^{4}$ ALBA Synchrotron Light Facility, Crta. BP 1413, Km. 3.3, 08290 Cerdanyola del Vallés, Barcelona, Spain \\ ${ }^{5}$ Research Institute for Interdisciplinary Science (RIIS), Okayama University, Okayama 700-8530, Japan \\ ${ }^{6}$ Department of Physics, Tokyo Metropolitan University, 1-1, Minami-osawa, Hachioji 192-0397, Japan \\ ${ }^{7}$ Department of Applied Physics, Waseda University, Tokyo 169-8555, Japan
}

(Received 3 March 2020; revised manuscript received 17 May 2020; accepted 12 June 2020; published 30 June 2020)

\begin{abstract}
The $\mathrm{BiS}_{2}$-based layered materials are characterized by a highly susceptible physical state, revealing a large response to external conditions. A particular case is the $\mathrm{EuFBiS}_{2}$ compound, showing a superconducting transition temperature $T_{c} \sim 0.3 \mathrm{~K}$ at ambient pressure. Upon increasing external pressure, $T_{c}$ goes through a large amplification, accompanied by a structural phase transition (SPT) from tetragonal to monoclinic symmetry. Here, we use a combination of $\mathrm{Eu} L_{3}$ - edge x-ray absorption spectroscopy and synchrotron x-ray diffraction to unveil the evolution of the Eu valence and lattice symmetry under high pressure. We find that the average Eu valence increases gradually with pressure, exhibiting a pressure plateau near the SPT, at which the $T_{c}$ increases sharply. Since in $\mathrm{EuFBiS}_{2}$ the charge carriers are introduced via self-doping induced by the mixed valence of the Eu ions, our findings clearly indicate that the role of the charge doping is marginal in the $T_{c}$ enhancement. On the other hand, the structural distortions, taking place at the SPT, play a central role in enhancing the superconducting properties of the $\mathrm{EuFBiS}_{2}$ system.
\end{abstract}

DOI: 10.1103/PhysRevB.101.214526

\section{INTRODUCTION}

Observation of superconductivity in $\mathrm{BiS}_{2}$-based layered compounds [1,2] stimulated intense experimental and theoretical efforts towards a thorough understanding of their physics and chemistry. These efforts have been further fueled by the discovery of their potential as thermoelectric materials [3,4]. Various $\mathrm{BiS}_{2}$-based systems have been synthesized and the most studied one is $\mathrm{RE}(\mathrm{O}, \mathrm{F}) \mathrm{BiS}_{2}(\mathrm{RE}=$ rare earth) that has tetragonal structure (Fig. 1) consisting of twin $\mathrm{BiS}_{2}$ layers alternated by REO layers [5,6]. Superconductivity in semiconducting REOBiS ${ }_{2}$ is generally introduced by substitution of $\mathrm{F}^{1-}$ for $\mathrm{O}^{2-}$, namely, doping electrons in the $\mathrm{Bi} 6 p_{x, y}$ orbitals at the Fermi level [7]. The highest superconducting transition temperature $T_{c}$ is $\sim 10 \mathrm{~K}$ in optimally doped $\mathrm{La}(\mathrm{O}, \mathrm{F}) \mathrm{BiS}_{2}[5,6]$ while the highest thermoelectric figure of merit, $z T$ is hosted by LaOBiSSe [3].

Soon after the discovery, it was recognized that the $\mathrm{BiS}_{2}$ lattice in these materials is intrinsically instable [8] and likely to consist of different local structure configurations $[9,10]$. Such an instability leads to a highly susceptible physical state that can be easily manipulated by physical or chemical pressures [5,6,11-13]. Indeed, the structural susceptibility has been well exploited to control superconductiviy and other physical properties of these materials by manipulating

\footnotetext{
*eugenio.paris@gmail.com

†naurang.saini@roma1.infn.it
}

misfit strain [14] between the crystal layers through chemical substitutions or by the application of hydrostatic pressure [5,6,11-13].

External substitution may not be always necessary for the electron doping in $\mathrm{BiS}_{2}$-based systems and it has been found that mixed valence of RE in the REO layer can provide electrons to the $\mathrm{BiS}_{2}$ layer through self-doping. This has been found recently in $\mathrm{CeOBiS}_{2}$ showing self-doped superconductivity without any external doping [15], albeit with lower $T_{c}$ than the optimum $T_{c}$ of the system. The self-doping appears to occur also in the isostructural $\mathrm{EuFBiS}_{2}$ [16] due to mixed valence of $\mathrm{Eu}\left(\mathrm{Eu}^{2+} / \mathrm{Eu}^{3+}\right)$, showing superconductivity below $\sim 0.3 \mathrm{~K}$. Similar self-doping occurs in multilayer $\mathrm{BiS}_{2}-$ based superconductors showing $T_{c} \sim 1.5 \mathrm{~K} \quad[17,18]$. Like other $\mathrm{BiS}_{2}$-based materials, self-doped systems are also highly susceptible to the external pressure. For example, EuFBiS ${ }_{2}$ reveals nearly a 30 times increase in $T_{c}$ under external pressure [19]. On the basis of high pressure studies, it has been argued that there are two superconducting phases, the ambient pressure phase with lower $T_{c}$ and the high pressure phase with higher $T_{c}$. These two phases have been associated with different structural symmetries as the system undergoes a structural phase transition from tetragonal to monoclinic upon external pressure. However, it has been highly debated if the monoclinic phase alone can be superconducting [20,21].

In mixed valence materials it is well known that a modification in the bond length may induce a change in the average valence state. Since the charge carriers in $\mathrm{EuFBiS}_{2}$ stem from self-doping induced by the valence fluctuation, the external 


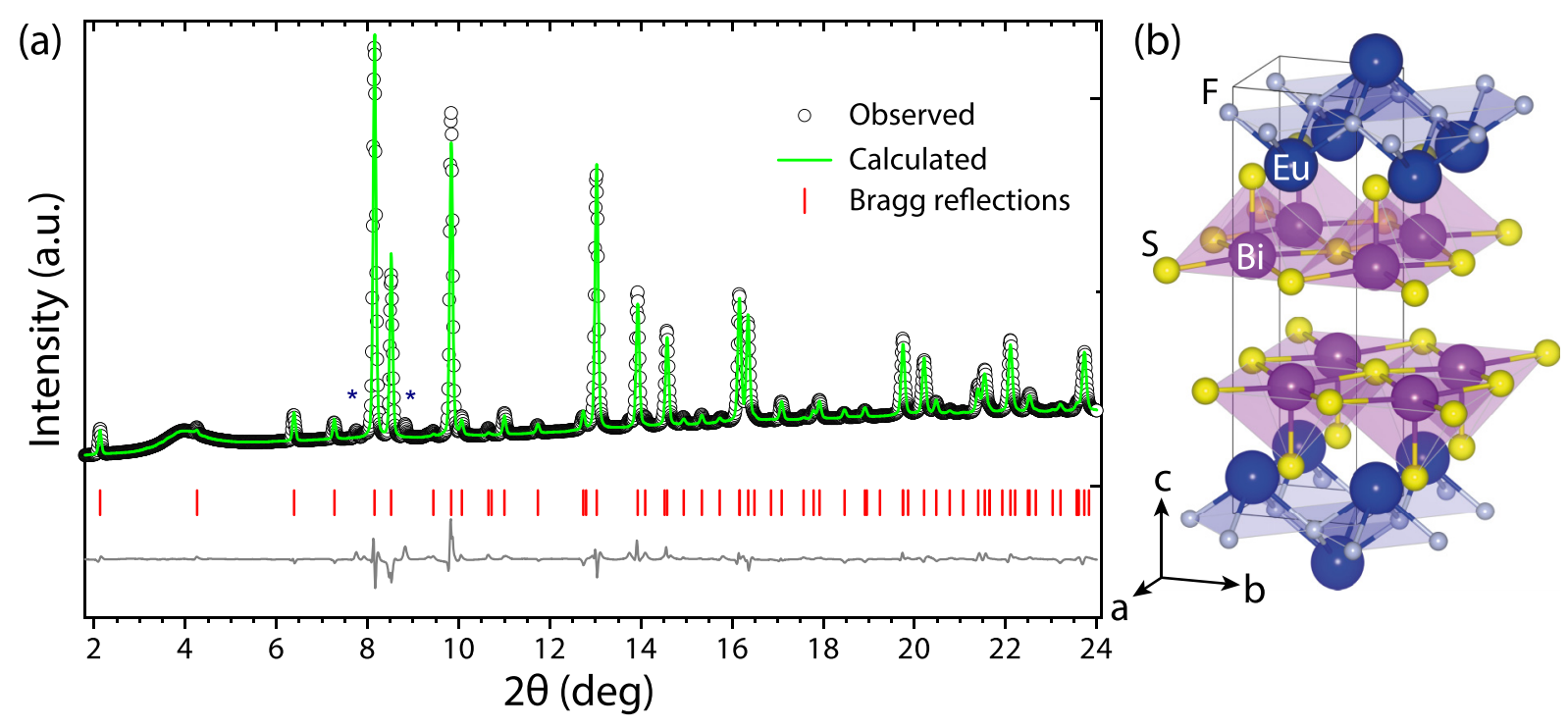

FIG. 1. (a) X-ray diffraction pattern of the studied EuFBiS ${ }_{2}$ sample at ambient conditions along with the Rietveld refinement results considering the tetragonal crystal structure. Bottom gray solid line corresponds to the difference curve. The impurity peaks are marked by *. (b) Tetragonal crystal structural model of $\mathrm{EuFBiS}_{2}$.

pressure is expected to tune the effective doping level. In this context, it is of central importance to investigate the role of pressure in tuning the density of states on one hand, and affecting the crystal symmetry on the other. Here, we have used $\mathrm{Eu} \mathrm{L}_{3}$-edge x-ray absorption spectroscopy (XAS) to provide a bulk-sensitive measurement of the average Eu valence state as a function of pressure. To provide insight on the evolution of the crystal structure, we have performed high-pressure x-ray diffraction (XRD) in the same pressure range. We find that the average valence of Eu increases gradually with a small plateau around the pressure at which the higher $T_{c}$ phase appears. In the same range of pressures, we have observed the coexistence of tetragonal and monoclinic phases, resulting from the firstorder structural phase transition, suggesting structural modifications to have important role. The results are discussed in the frame of interplay of self-doping and structural modification to control the granular superconductivity in $\mathrm{EuFBiS}_{2}$ system.

\section{EXPERIMENTAL DETAILS}

A conventional solid-state reaction method was used to prepare polycrystalline samples of $\mathrm{EuFBiS}_{2}$ [22]. Figure 1(a) shows ambient pressure XRD pattern of the EuFBiS 2 system used for the present study. The Rietveld refinement analysis shows that the ambient pressure crystal structure is tetragonal (Fig. 1) in agreement with that earlier reports $[19,22]$. A small impurity phase was identified as $\mathrm{Bi}_{2} \mathrm{~S}_{3}$ (estimated to be less than $1 \%$ ).

The XAS measurements were performed at the energy dispersive extended $\mathrm{x}$-ray-absorption fine structure (EXAFS) beamline ID24 [23] of the European Synchrotron Radiation Facility (ESRF), Grenoble. The x-ray source consisted of two undulators whose gaps were adjusted to tune the maximum of the first harmonic for $\mathrm{x}$-ray energies around the Eu $\mathrm{L}_{3}$-edge. The horizontal focusing was set by a curved (Si 111) crystal polychromator while a bent Si mirror was used for the vertical focusing and higher harmonic rejection.
The focused beam size on the sample was of $30 \times 30 \mu \mathrm{m}^{2}$ (full width half maximum). The spectra were recorded in transmission mode using a FreLon CCD camera detector [24]. The energy calibration was done using a reference standard sample at ambient conditions. Membrane driven opposingplate diamond anvil cells (DACs) equipped with $600-\mu \mathrm{m}$ culet I-A diamonds were used to pressurize $\mathrm{EuFBiS}_{2}$ samples, with silicone oil [25] as a pressure transmitting medium. The gaskets were made of a $250-\mu \mathrm{m}$-thick stainless steel foil with a sample chamber of $\sim 180-\mu \mathrm{m}$-diameter and 60 $\mu \mathrm{m}$ height. The pressure was measured in situ, exploiting the standard ruby fluorescence technique [26]. High pressure XRD measurements were performed using wavelength $\lambda=$ $0.5 \AA$ at the Xpress beam line [27] of the Elettra Synchrotron in Trieste at which a MAR345 image plate detector is used to acquire the diffraction data. A gear-driven Boehler-Almax plate DAC with large $\mathrm{x}$-ray opening, containing diamonds of culet size $600 \mu \mathrm{m}$ was used for the high-pressure XRD measurements. A $200-\mu$ m-thick stainless steel gasket, with a sample chamber of $200-\mu \mathrm{m}$ diameter and $70-\mu \mathrm{m}$ height was used. The pressure was measured by monitoring the diffraction pattern from a reference $\mathrm{Cu}$ metal foil. For both the XAS and XRD experiments, silicone oil was also used as the pressure transmitting medium. The silicone oil was adopted as hydrostatic medium due to its suitability for sample loadings in the DAC (ensuring lower sample volume in the chamber compared to pressure transmitting medium) and its sufficient hydrostatic properties in the low pressure interval used in the present work.

\section{RESULTS AND DISCUSSION}

Figure 2 shows Eu $\mathrm{L}_{3}$ - edge XAS spectra measured on EuFBiS $_{2}$ at several pressures. The spectra are described by Eu $2 \mathrm{p}_{3 / 2} \rightarrow 5 d$ (or $2 \mathrm{p}_{3 / 2} \rightarrow 6 s$ ) transition in which the dipole selection rule $(l= \pm 1)$ permits us to probe empty $5 d$ and admixed $4 f$ states considering that the probability of $2 \mathrm{p}_{3 / 2} \rightarrow$ 

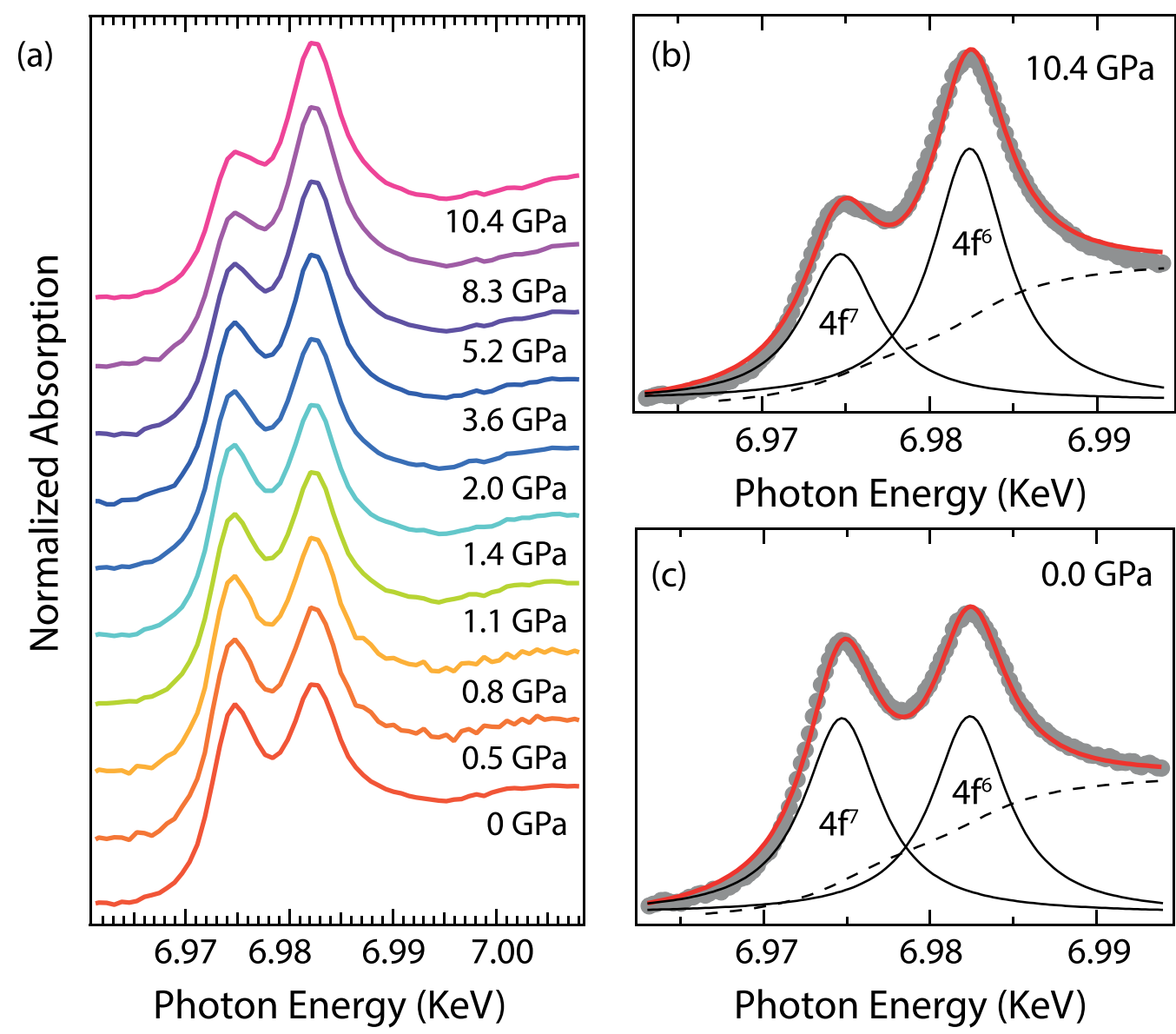

FIG. 2. (a) Normalized $\mathrm{Eu} \mathrm{L}_{3}$-edge x-ray absorption spectra of EuFBiS ${ }_{2}$ at different pressures. (b), (c) XAS spectra for $\mathrm{P}=0$ and $10.4 \mathrm{GPa}$ are shown with deconvoluted Lorentzian functions used to estimate the two valence states of Eu, i.e., Eu ${ }^{2+}$ and Eu ${ }^{3+}$. The total background representing the edge step, sum of two arctangent functions, is shown with a dashed line.

$6 s$ is relatively small and can be ignored. The XAS spectra exhibit a white line like transition that is split into two peaks at $\sim 6975 \mathrm{eV}$ and at $\sim 6983 \mathrm{eV}$. These peaks correspond to the admixed $4 f^{7}$ and $4 f^{6}$ final states and indicate the mixed valence state in $\mathrm{Eu}^{2+}$ and $\mathrm{Eu}^{3+}[28,29]$, consistent with earlier studies [16,30].

It is possible to quantify the average valence of Eu by deconvolution of the $\mathrm{Eu} \mathrm{L}_{3}$-edge XAS spectra. In particular, we have carried out the XAS deconvolution using two spectral components to account for the $\mathrm{Eu}^{2+}$ and $\mathrm{Eu}^{3+}$ contributions. Each component consists of a Lorentzian function (to model the white line) and an arctangent function (to model the absorption edge jump). Figures 2(b) and 2(c) show typical deconvolution results. The two Lorentzian components are shown as black solid lines, while the dashed line represents the total background, i.e., the sum of the two arctangent contributions. The Eu valence is determined from the relative spectral weight of the $\mathrm{Eu}^{2+}$ and $\mathrm{Eu}^{3+}$ components. We have used this method to track the changes in the valence state, while the absolute value of the valence at ambient pressure is calibrated by repeating the XAS measurement on the sample outside of the pressure cell. The evolution of the Eu valence, obtained using the above method, is shown in Fig. 3 as a function of pressure. The average valence increases gradually with external pressure and reaches a small plateau at $\sim 1 \mathrm{GPa}$. The pressure dependence of the superconducting transition temperature of EuFBiS $S_{2}$ is also shown in the zoomover. As appears, the superconducting transition temperature of the system increases sharply around this pressure plateau. It is worth recalling that similar pressure induced valence plateau has been observed [31-33] in several rare-earth monochalcogenides and assigned to the multichannel Kondo effect [31]. We will come back again to comment on this later.

It is interesting to note that the average valence of $\mathrm{Eu}$ continues to increase and therefore, average self-doping is expected to increase as well. On the other hand, the $T_{c}$ does not change after the sharp increase at $\sim 1 \mathrm{GPa}$ suggesting that the superconductivity in $\mathrm{EuFBiS}_{2}$ at higher $T_{c}$ may not be strictly dependent on the doping. In addition, it is also possible that not all the available charge is transferred to the $\mathrm{BiS}_{2}$ layer and a part of it may be getting localized. This would be consistent with angle resolved photoemission measurements revealing that the electron density at $\mathrm{E}_{F}$ is generally less than what is doped, commonly found in different families of the $\mathrm{BiS}_{2}$ based superconducting systems [34-37]. At this point it would be interesting to see how the structure of $\mathrm{EuFBiS}_{2}$ behaves under external pressure.

Figure 4 shows the XRD patterns of $\mathrm{EuFBiS}_{2}$ at various pressures. At pressure above $\sim 1 \mathrm{GPa}$ the diffraction pattern changes due to change in the average structural symmetry from tetragonal $(P 4 / \mathrm{nmm})$ to monoclinic $\left(P 2_{1} / \mathrm{m}\right)$, consistent with earlier reports on the same system [19] and on 


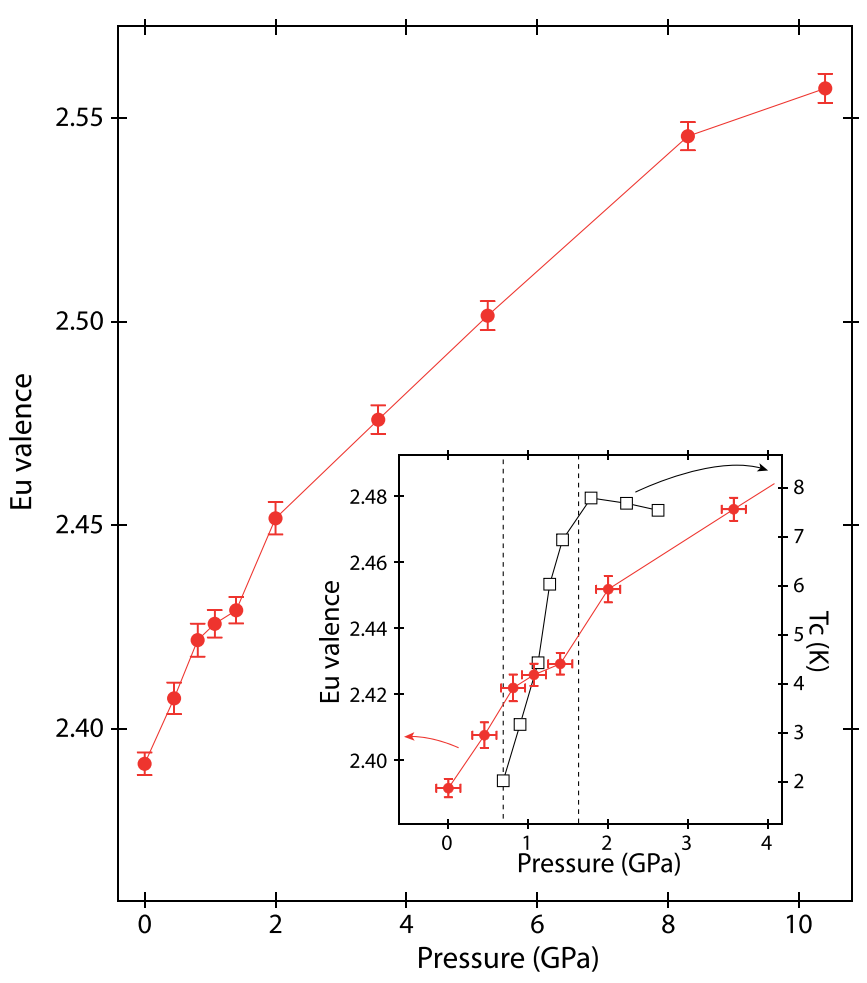

FIG. 3. Eu valence estimated from XAS is shown as a function of pressure for $\mathrm{EuFBiS}_{2}$. The change in the superconducting transition temperature is also plotted together with a zoomover.

other $\mathrm{BiS}_{2}$-based materials $[6,20,21]$. Here, the structural phase transition seems to be of first order with a region of coexistence. The transition takes place in the range of pressure in which the system shows a sharp increase in $T_{c}$ and the $\mathrm{Eu}$ valence plateau appears, suggesting this behavior is likely to be induced by the phase coexistence. It is worth mentioning that we have observed a small broadening in the XRD peaks under pressure (comparable to the one observed in similar systems in monoclinic phase $[19,21]$ ), that should be intrinsic to the high-pressure phase of $\mathrm{EuFBiS}_{2}$ rather than due to any nonhydrostatic nature of the pressure medium used in this work.

Figure 5 shows the evolution of the lattice parameters as a function of pressure. The Rietveld analysis of the diffraction data permitted us to extract the lattice parameters of the two phases. The lattice parameters indicate a unit-cell volume discontinuity going from the low pressure tetragonal phase to the high pressure monoclinic phase [Fig. 5(d)]. Incidentally, several monochalcogenides show similar change is the valence state and unit-cell volume discontinuity [31-33], i.e., a valence plateau where the unit-cell volume discontinuity appears without a change in the crystal symmetry. Such a correlation was assigned to a possible multichannel Kondo effect. However, unlike earlier observations on the monochalcogenides, EuFBiS 2 reveals a clear change in the crystal structure symmetry with a weak pressure induced discontinuity in unit-cell volume. From the pressure evolution of the lattice parameters as well the angle $\beta$, the narrow pressure region of $0.8-1.8 \mathrm{GPa}$ can be tentatively assigned to the coexistence region. We should mention that the present analysis does not provide a reasonable estimate of the relative
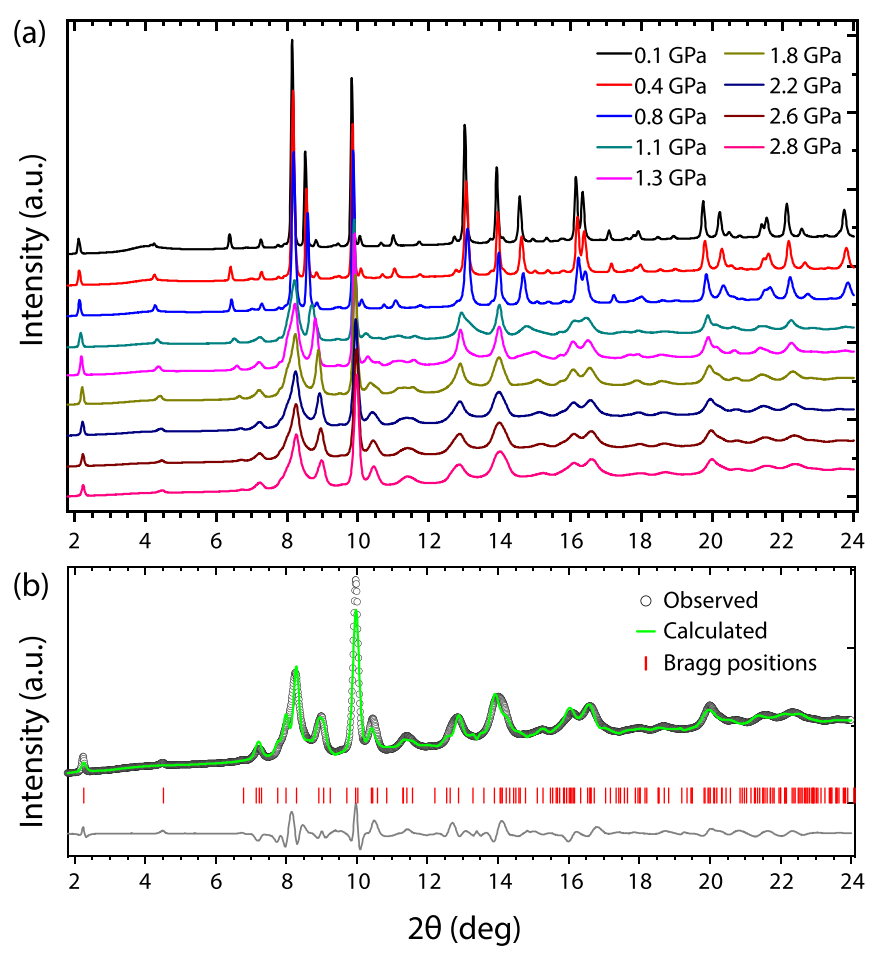

FIG. 4. (a) Pressure dependence of the x-ray diffraction patterns of EuFBiS ${ }_{2}$. Data taken at different pressures is shown with a vertical shift for better clarity. (b) X-ray diffraction pattern of EuFBiS $\mathrm{St}_{2}$ at $\mathrm{P}=3.2 \mathrm{GPa}$, along with the Rietveld refinement profile relative to the high-pressure monoclinic phase.

fraction of different phases in the narrow pressure regime of coexistence due to the strong correlation between the fit parameters when two phases are simultaneously considered to describe the observed experimental pattern. Interestingly, the observed pressure induced unit-cell volume discontinuity coincides with a large increase in the superconducting transition temperature of the system. Indeed, the $T_{c}$ increases almost 30 times and saturates beyond the pressure region at which the structural phase transition (SPT) takes place. The transition temperature hardly shows any change beyond the pressure of coexistence, while the average structural symmetry turns to monoclinic.

Let us attempt to understand the above results in the light of known facts about $\mathrm{BiS}_{2}$-based materials. On the one hand it is known that undoped $\mathrm{LaOBiS}_{2}$ system goes through a SPT from the tetragonal to the monoclinic phase $[20,21]$ and remains nonsuperconducting even at very high pressure. On the other hand, if the system is doped and put under pressure, there is a transition from the tetragonal to the monoclinic phase accompanied by superconductivity at higher $T_{c}[5,6,11-$ 13]. Therefore, neither the doping alone nor the structural instability alone can induce superconductivity in $\mathrm{LaOBiS}_{2}$ and both of the optimized host lattice and doping seem important for the higher $T_{c}$ phase in these materials. These findings lead to the question of why the high-pressure low-symmetry phase enhances superconductivity in this class of materials.

The $\mathrm{BiS}_{2}$-based materials are characterized by a local structure instability $[9,10]$ that can be described by different in-plane (Bi-S1) bond distances $[38,39]$ together with an intrinsically short axial (Bi-S2) bond. Such a local structure 

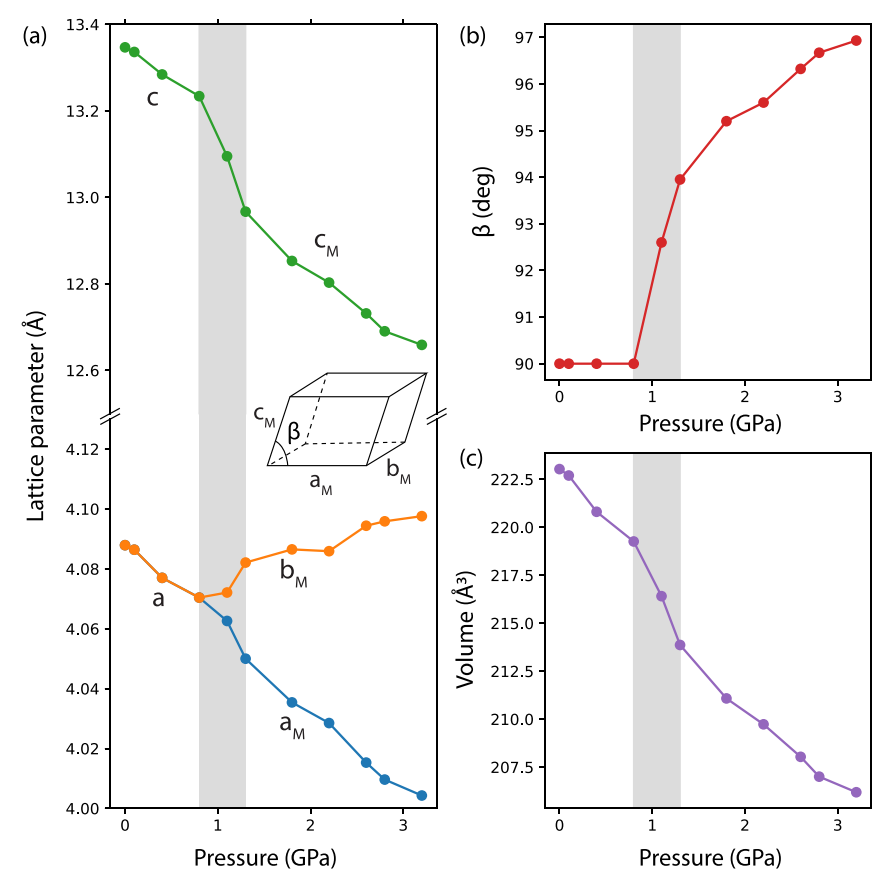

FIG. 5. Lattice parameters (a), angle (b), and unit cell volume (c) plotted as a function of pressure for $\mathrm{EuFBiS}_{2}$. The inset of panel (a) depicts the monoclinic unit cell. The gray area in each panel shows the region of phase coexistence marking the structural phase transition.

configuration is driven by Bi defect chemistry (asymmetric charge distribution on $\mathrm{Bi}$ ) associated with the coupling of $\mathrm{Bi}$ 6s - Bi 6p states due to Bi 6s - S 3p interaction, common to different active lone-pair systems [40-42]. In this configuration, only a part of the doped charge remains itinerant, i.e., transferred to the $\mathrm{Bi} 6 \mathrm{p}_{x . y}$ orbitals [34-37], while the rest remains localized in local structure distortions $[34,43]$. Chemical pressure by Se substitution for $\mathrm{S}$ in the structure reduces the structural distortions [39] rendering the local structure configuration more symmetric [40]. Therefore, one can expect a similar mechanism being active upon the application of external pressure $[41,42]$ also in the present case. However, in this picture a gradual change is expected unlike the observed pressure induced abrupt variation in $T_{c}$. This indicates some additional mechanism in which the SPT may have some important role. Considering the earlier studies we think that the abrupt increase in $T_{c}$ is related with the granularity of $\mathrm{BiS}_{2}$-based materials due to their local structure instability. For example, the self-doped $\mathrm{CeOBiS}_{2}$ system with mixed valence Ce menifests electronic phase separation at ambient pressure [44] showing metallic grains embedded in a majority non-metallic phase. Similarly, self-doped $\mathrm{EuFBiS}_{2}$ shows an inhomogeneous charge distribution [45] at ambient pressure. The metallic and non-metallic phases are characterized by different local structure configurations having direct effect on the Fermi surface properties $[34,44,45]$. At the pressure induced SPT the local geometry involving the in-plane (Bi-S1) and axial (Bi-S2) bond distances are likely to get renormalized resulting in increased metallic grains. Thus, $\mathrm{EuFBiS}_{2}$ at ambient pressure with small density of conducting grains (and hence limited phase coherence) $[46,47]$ is in the low $T_{c}$ phase. Under external pressure, more itinerant charge is available at the structural phase transition due to the modified local structure configuration with larger metallic grain connectivity (and hence small phase fluctuations) driving the system to the higher $T_{c}$ phase. Further pressure hardly affects the superconductivity due to optimum phase coherence resulting in highest possible $T_{c}$.

\section{CONCLUSIONS}

In summary, we have studied the evolution of the selfdoping and the lattice structure in $\mathrm{EuFBiS}_{2}$ as a function of pressure. At $\sim 1 \mathrm{GPa}$, the crystal structure transforms from tetragonal (at low pressure) to monoclinic (at high pressure), showing a region of coexistence of the two crystal phases in which $T_{c}$ increases sharply. On the other hand, the Eu valence, and hence the self-doping, increases continuously over a large pressure range encompassing the two structural phases. Therefore, the lattice degree of freedom, rather than the charge doping, largely controls the superconducting properties. In the low-pressure tetragonal phase, it was previously reported that the doped charges are trapped within local lattice distortions, resulting in a lower effective doping and a granular superconducting state with low $T_{c}$. In the high-pressure phase, with average monoclinic structure, the local Bi-S bond network is modified. Since such local parameters control the granularity and the electron mobility in this class of materials, their evolution is probably responsible for the large enhancement of $T_{c}$. Future high-pressure experiments using local structural probes (such as EXAFS) would be beneficial to resolve the evolution of the local structure.

\section{ACKNOWLEDGMENTS}

The authors thank ESRF and Elettra for beamtime allocation and ID-24 and XPress beamline staffs for excellent technical support during the measurements. B.J. acknowledges IISc Bangalore and ICTP Trieste for the IISc-ICTP fellowship. The work is a part of the executive protocol of the general agreement for cooperation between the Sapienza University of Rome, the Tokyo Metropolitan University, and Okayama University including the Waseda University, Japan.
[1] Y. Mizuguchi, H. Fujihisa, Y. Gotoh, K. Suzuki, H. Usui, K. Kuroki, S. Demura, Y. Takano, H. Izawa, and O. Miura, Phys. Rev. B 86, 220510(R) (2012).

[2] Y. Mizuguchi, S. Demura, K. Deguchi, Y. Takano, H. Fujihisa, Y. Gotoh, H. Izawa, and O. Miura, J. Phys. Soc. Jpn. 81, 114725 (2012).
[3] Y. Mizuguchi, A. Nishida, A. Omachi, and O. Miura, Cogent Phys. 3, 1156281 (2016).

[4] A. Omachi, J. Kajitani, T. Hiroi, O. Miura, and Y. Mizuguchi, J. Appl. Phys. 115, 083909 (2014).

[5] Y. Mizuguchi, J. Phys. Chem. Solids 84, 34 (2015). 
[6] D. Yazici, I. Jeon, B. D. White, and M. B. Maple, Physica C 514, 218 (2015).

[7] H. Usui, K. Suzuki, and K. Kuroki, Phys. Rev. B 86, 220501(R) (2012).

[8] T. Yildirim, Phys. Rev. B 87, 020506(R) (2013).

[9] Q. Liu, X. Zhang, and A. Zunger, Phys. Rev. B 93, 174119 (2016).

[10] X. Zhou, Q. Liu, J. A. Waugh, H. Li, T. Nummy, X. Zhang, X. Zhu, G. Cao, A. Zunger, and D. S. Dessau, Phys. Rev. B 95, 075118 (2017).

[11] R. Jha, H. Kishan, and V. P. S. Awana, J. Phys. Chem. Solids 84, 17 (2015).

[12] Y. Fang, D. Yazici, B. D. White, and M. B. Maple, Phys. Rev. B 92, 094507 (2015).

[13] C. T. Wolowiec, B. D. White, I. Jeon, D. Yazici, K. Huang, and M. B. Maple, J. Phys.: Condens. Matter 25, 422201 (2013).

[14] N. Poccia, A. Ricci, and A. Bianconi, Adv. Condens. Matter Phys. 2010, 261849 (2010)

[15] M. Nagao, A. Miura, I. Ueta, S. Watauchi, and I. Tanaka, Solid State Commun. 245, 11 (2016).

[16] H. F. Zhai, Z. T. Tang, H. Jiang, K. Xu, K. Zhang, P. Zhang, J. K. Bao, Y. L. Sun, W. H. Jiao, I. Nowik, I. Felner, Y. K. Li, X. F. Xu, Q. Tao, C. M. Feng, Z. A. Xu, and G. H. Cao, Phys. Rev. B 90, 064518 (2014).

[17] H. F. Zhai, P. Zhang, S. Q. Wu, C. Y. He, Z. T. Tang, H. Jiang, Y. L. Sun, J. K. Bao, I. Nowik, I. Felner, Y. W. Zeng, Y. K. Li, X. F. Xu, Q. Tao, Z. A. Xu, and G. H. Cao, J. Am. Chem. Soc. 136, 15386 (2014).

[18] Z. Haque, G. S. Thakur, R. Parthasarathy, B. Gerke, T. Block, L. Heletta, R. Pöttgen, A. G. Joshi, G. K. Selvan, S. Arumugam, L. C. Gupta, and A. K. Ganguli, Inorg. Chem. 56, 3182 (2017).

[19] C. Y. Guo, Y. Chen, M. Smidman, S. A. Chen, W. B. Jiang, H. F. Zhai, Y. F. Wang, G. H. Cao, J. M. Chen, X. Lu, and H. Q. Yuan, Phys. Rev. B 91, 214512 (2015).

[20] R. Sagayama, H. Sagayama, R. Kumai, Y. Murakami, T. Asano, J. Kajitani, R. Higashinaka, T. D. Matsuda, and Y. Aoki, J. Phys. Soc. Jpn. 84, 123703 (2015).

[21] T. Tomita, M. Ebata, H. Soeda, H. Takahashi, H. Fujihisa, Y. Gotoh, Y. Mizuguchi, H. Izawa, O. Miura, S. Demura, K. Deguchi, and Y. Takano, J. Phys. Soc. Jpn. 83, 063704 (2014).

[22] G. Jinno, R. Jha, A. Yamada, R. Higashinaka, T. D. Matsuda, Y. Aoki, M. Nagao, O. Miura, and Y. Mizuguchi, J. Phys. Soc. Jpn. 85, 124708 (2016).

[23] S. Pascarelli, O. Mathon, T. Mairs, I. Kantor, G. Agostini, C. Strohm, S. Pasternak, F. Perrin, G. Berruyer, P. Chappelet, C. Clavel, and M. C. Dominguez, J. Synchrotron Radiat. 23, 353 (2016).

[24] J. C. Labiche, O. Mathon, S. Pascarelli, M. A. Newton, G. G. Ferre, C. Curfs, G. Vaughan, A. Homs, and D. D. Carreiras, Rev. Sci. Instrum. 78, 091301 (2007).

[25] Y. Shen, R. S. Kumar, M. Pravica, and M. F. Nicol, Rev. Sci. Instrum. 75, 4450 (2004).

[26] H. K. Mao, P. M. Bell, J. W. Shaner, and D. J. Steinberg, J. Appl. Phys. 49, 3276 (1978).

[27] P. Lotti, S. Milani, M. Merlini, B. Joseph, F. Alabarse, and A. Lausi, J. Synchrotron Radiat. 27, 222 (2020).
[28] H. Wada, A. Nakamura, A. Mitsuda, M. Shiga, T. Tanaka, H. Mitamura, and T. Goto, J. Phys.: Condens. Matter 9, 7913 (1997).

[29] A. P. Menushenkov, A. A. Yaroslavtsev, A. Y. Geondzhian, R. V. Chernikov, Y. V. Zubavichus, X. Tan, and M. Shatruk, J. Supercond. Nov. Mag. 28, 995 (2015).

[30] Y. Mizuguchi, E. Paris, T. Wakita, G. Jinno, A. Puri, K. Terashima, B. Joseph, O. Miura, T. Yokoya, and N. L. Saini, Phys. Rev. B 95, 064515 (2017).

[31] I. Jarrige, J.-P. Rueff, S. R. Shieh, M. Taguchi, Y. Ohishi, T. Matsumura, C.-P. Wang, H. Ishii, N. Hiraoka, and Y. Q. Cai, Phys. Rev. Lett. 101, 127401 (2008).

[32] I. Jarrige, H. Yamaoka, J.-P. Rueff, J.-F. Lin, M. Taguchi, N. Hiraoka, H. Ishii, K. D. Tsuei, K. Imura, T. Matsumura, A. Ochiai, H. S. Suzuki, and A. Kotani, Phys. Rev. B 87, 115107 (2013).

[33] B. Joseph, R. Torchio, C. Benndorf, T. Irifune, T. Shinmei, R. Pöttgen, and A. Zerr, Phys. Chem. Chem. Phys. 19, 17526 (2017).

[34] T. Sugimoto, D. Ootsuki, E. Paris, A. Iadecola, M. Salome, E. F. Schwier, H. Iwasawa, K. Shimada, T. Asano, R. Higashinaka, T. D. Matsuda, Y. Aoki, N. L. Saini, and T. Mizokawa, Phys. Rev. B 94, 081106(R) (2016).

[35] Z. R. Ye, H. F. Yang, D. W. Shen, J. Jiang, X. H. Niu, D. L. Feng, Y. P. Du, X. G. Wan, J. Z. Liu, X. Y. Zhu, H. H. Wen, and M. H. Jiang, Phys. Rev. B 90, 045116 (2014).

[36] L. K. Zeng, X. B. Wang, J. Ma, P. Richard, S. M. Nie, H. M. Weng, N. L. Wang, Z. Wang, T. Qian, and H. Ding, Phys. Rev. B 90, 054512 (2014).

[37] E. Paris, T. Sugimoto, T. Wakita, A. Barinov, K. Terashima, V. Kandyba, O. Proux, J. Kajitani, R. Higashinaka, T. D. Matsuda, Y. Aoki, T. Yokoya, T. Mizokawa, and N. L. Saini, Phys. Rev. B 95, 035152 (2017).

[38] A. Athauda, J. Yang, S. Lee, Y. Mizuguchi, K. Deguchi, Y. Takano, O. Miura, and D. Louca, Phys. Rev. B 91, 144112 (2015).

[39] E. Paris, Y. Mizuguchi, T. Wakita, K. Terashima, T. Yokoya, T. Mizokawa, and N. L. Saini, J. Phys.: Condens. Matter 30, 455703 (2018).

[40] For a brief review, see, e.g., A. Walsh, D. J. Payne, R. G. Egdell, and G. W. Watson, Chem. Soc. Rev. 40, 4455 (2011).

[41] L. Arnskov Olsen, J. Lopez-Solano, A. Garcia, T. Balic-Zunic, and E. Makovicky, J. Solid State Chem. 183, 2133 (2010).

[42] L. F. Lundegaard, E. Makovicky, T. Boffa-Ballaran, and T. Balic-Zunic, Phys. Chem. Miner. 32, 578 (2005).

[43] T. Sugimoto, B. Joseph, E. Paris, A. Iadecola, T. Mizokawa, S. Demura, Y. Mizuguchi, Y. Takano, and N. L. Saini, Phys. Rev. B 89, 201117(R) (2014).

[44] T. Sugimoto, E. Paris, T. Wakita, K. Terashima, T. Yokoya, A. Barinov, J. Kajitani, R. Higashinaka, T. D. Matsuda, Y. Aoki, T. Mizokawa, and N. L. Saini, Sci. Rep. 8, 2011 (2018).

[45] T. Sugimoto, E. Paris, K. Terashima, A. Barinov, A. Giampietri, T. Wakita, T. Yokoya, J. Kajitani, R. Higashinaka, T. D. Matsuda, Y. Aoki, T. Mizokawa, and N. L. Saini, Phys. Rev. B 100, 064520 (2019).

[46] I. S. Beloborodov, A. V. Lopatin, V. M. Vinokur, and K. B. Efetov, Rev. Mod. Phys. 79, 469 (2007).

[47] P. Brighi, M. Grilli, B. Leridon, and S. Caprara, Phys. Rev. B 100, 174518 (2019). 